\title{
La dynamique de la discrimination salariale et de l'équité gendorielle sur le marché du travail au Maroc
}

Sami Zouari

Follow this and additional works at: https://knowledgecommons.popcouncil.org/departments_sbsr-pgy

Part of the Demography, Population, and Ecology Commons, Family, Life Course, and Society Commons, Gender and Sexuality Commons, International Public Health Commons, Labor Economics Commons, and the Work, Economy and Organizations Commons How does access to this work benefit you? Let us know!

\section{Recommended Citation}

Zouari, Sami. 2011. "La dynamique de la discrimination salariale et de l'équité gendorielle sur le marché du travail au Maroc," Gender and Work in the MENA Region Working Paper no. 20. Cairo: Population Council. 


\section{NUMBER 20}

JUNE 2011

GENDER AND WORK IN THE MENA REGION

WORKING PAPER SERIES

Poverty, Job Quality and Labor Market Dynamics

La dynamique de la discrimination

salariale et de l'équité gendorielle

sur le marché du travail au Maroc

Sami Zouari

(2 Population Council 
Sami Zouari, Economiste, Institut Supérieur de Gestion Industrielle de Sfax, Sfax, Tunsie. E-mail : sami.zouari@isgis.rnu.tn

Ce document traite de projets appuyé par le Centre de recherches pour le développement international, organism canadien (www.crdi.ca).

\section{IDRC * CRDI}

Canadà

\section{Population Council}

Research that makes a difference

Le Population Council s'attelle aux questions cruciales de santé et de développement -qu'il s'agisse d'endiguer la propagation du VIH, d'améliorer la santé reproductive et d'assurer que les jeunes mènent une vie satisfaisante et productive. Grâce à des travaux de recherche en biomédecine, en sciences sociales et en santé publique dans 50 pays, le Population Council travaille avec

ses partenaires pour offrir des solutions qui conduisent à des politiques, des programmes et des technologies plus efficaces pour améliorer la vie partout dans le monde. Fondé en 1952 et basé à New York, le Population Council est une organisation non gouvernementale à but non lucratif avec un conseil d'administration international.

Population Council

Egypt Office

59 Misr Helwan Agricultural Road, Maadi, Cairo, Egypt

Tel.: (+202) 2525-5965, (+202) 2525-5967, (+202) 2525-5968

Facsimile: (+202) 2525-5962

Website: http://www.popcouncil.org

E-mail: pcouncil@popcouncil.org

(c) 2011 The Population Council, Inc.

Toute partie de ce document peut être reproduite en quantité limitée sans la permission du Population Council pourvu qu'elle soit distribuée gratuitement ou à des fins non lucrative et que la source soit identifiée. Le Population Council voudraient bien recevoir une copie de tous les matériaux qui emploient le texte.

ISSN: $11873 / 2011$ 
NUMBER 20

JUNE 2011

GENDER AND WORK IN THE MENA REGION WORKING PAPER SERIES

Poverty, Job Quality and Labor Market Dynamics

\section{La dynamique de la discrimination salariale et de l'équité gendorielle sur le marché du travail au Maroc}

Sami Zouari 



\section{Table of contents}

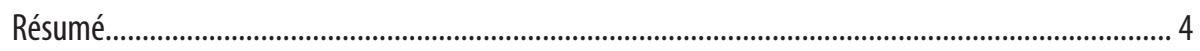

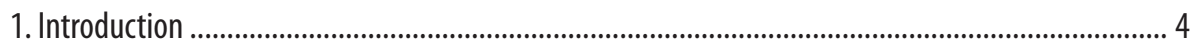

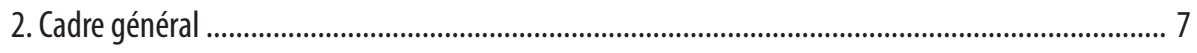

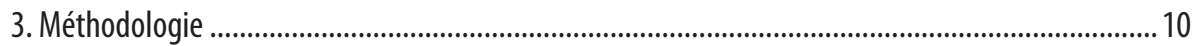

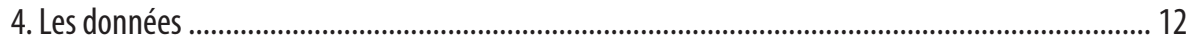

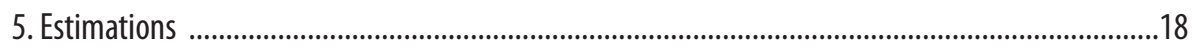

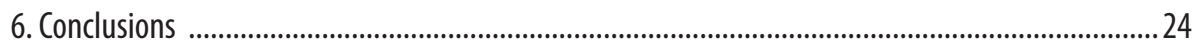

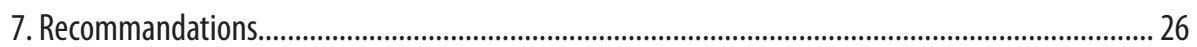

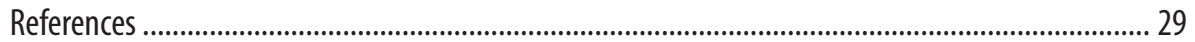




\section{Résumé}

La présente analyse s'est interrogée sur l'écart salarial entre les hommes et les femmes au Maroc. Il a été conclu que l'écart de salaire entre les hommes et les femmes au Maroc s'était réduit de peu dans le privé mais considérablement dans le public. Nous avons utilisé les méthodes de décompositions d'Oaxaca et Ransom et celle de Neumann et Oaxaca. Les estimations ont révélé que les femmes sont rémunérées de 34,6\% et $30,9 \%$ moins que les hommes et ce successivement en I991 et 1999. $35,7 \%$ et $29,4 \%$ de ces écarts sont inexpliqués. Ces taux traduisent ainsi la discrimination à l'égard des femmes. Par ailleurs, les différences des caractéristiques expliquent quant à elles, la présence de I, $5 \%$ de l'écart en I999 alors qu'il était carrément négatif en 199I indiquant ainsi que l'écart opère en faveur des femmes.

Par ailleurs, il a été souligné l'importance de l'éducation des femmes dans le secteur public. En effet, avec un écart salarial avoisinant 31,5\% en I99I et 2I,8\% en I999 dans le secteur public, nous avions pu dégager une part assez significative en termes d'écart justifié en faveur des femmes. Cet écart qui était égal à $-5,4 \%$ en I99I est passé à -I3,I\% en I999. Le signe négatif indique un avantage féminin reflétant ainsi l'avancée des femmes en termes d'éducation comme nous l'avons précédemment souligné.

Néanmoins, dans le secteur privé, l'écart salarial homme-femme était assez important puisqu'en I99I il était de l'ordre de 37,5\% pour passer en 1999 à 33,5\%. Cette baisse, relativement faible comparée à celle du secteur public, bien qu'elle soit accompagnée par une augmentation de l'écart justifié qui est passé de I à $6 \%$, elle a été due essentiellement à la diminution de l'écart injustifié qui est passé de $36,7 \%$ à $27,6 \%$.

\section{Introduction}

Deux travailleurs qui ont les mêmes caractéristiques et la même productivité devraient être également rémunérés quelque soient leurs genres et leurs races. Cependant, sur le marché du travail, à productivité égale, l'homme devance la femme à la rémunération: c'est ce qu'on appelle la discrimination salariale. Ce problème a toujours suscité un intérêt inéluctable. Nombreuses études ont mis en évidence l'existence d'une discrimination salariale par genre et ont proposé des politiques économiques pour contribuer à l'apaisement de ce phénomène. 
Comment expliquer, qu'à productivité égale, les hommes et les femmes perçoivent des salaires différents? Plusieurs approches théoriques ont été avancées. La première catégorie de modèles se place dans un cadre néoclassique dans lequel les employeurs connaissent parfaitement la productivité des individus, mais discriminent par goût [Becker (1957)]. Selon cette théorie, les employeurs font partie d'un groupe majoritaire discriminant (hommes) et ressentent un déplaisir éventuel lors de tout contact avec des individus du camp des minorités discriminées (les individus du sexe opposé). Ce goût entraîne une désutilité à l'embauche des femmes, et augmente par conséquent, leurs coûts d'emploi. Les femmes reçoivent donc un salaire plus faible que les hommes pour compenser le préjudice moral que procure leur embauche.

Dans la deuxième catégorie de modèle, les employeurs discriminent parce qu'ils n'observent qu'imparfaitement la productivité des femmes. Il s'agit d'une discrimination non intentionnelle dite statistique, résultante d'une information imparfaite sur le marché du travail [Phelps (1972); Arrow (1973)]. En effet, lors du recrutement, les employeurs, n'observent qu'imparfaitement la productivité instantanée, s'ils considèrent qu'en moyenne les travailleurs hommes sont plus performants, plus qualifiés, que les travailleurs femmes, ils vont rationnellement préférer embaucher un homme. Il en résulte une discrimination statistique à l'encontre des femmes qui provient d'une estimation d'une donnée non observable "la productivité" à partir d'une donnée observable "le genre". Pour estimer la productivité d'un candidat, les employeurs se fondent alors sur leur croyance concernant la moyenne et la dispersion de la productivité au sein du groupe auquel appartient le candidat (groupe de femmes ou groupe d'hommes). Ainsi, deux candidats qui ont les mêmes caractéristiques observables peuvent recevoir des salaires différents parce que la productivité moyenne de leurs groupes respectifs n'est pas la même.

Dans d'autres versions de ces modèles (Lazear et Rosen (I990)), c'est l'attachement futur des femmes au marché de travail qui n'est pas observable au moment de l'embauche et non pas leurs productivités. Les employeurs anticipent l'absence fréquente des femmes de la sphère productive pour la sphère domestique et leur exigent des capacités supérieures à celles des hommes afin de compenser leur forte probabilité d'abandon. Le faible attachement des femmes au marché du travail augmente également le coût de leur promotion: l'employeur ne leur accorde une promotion que s'il est certain qu'elle ne quittera pas l'entreprise.

Sur le plan empirique, plusieurs travaux ont été élaborés pour mettre en évidence le problème de la discrimination salariale aussi bien aux USA qu'en Europe. Les études diffèrent par le choix de la population étudié 
(industrie, milieu académique, les diplômés d'une université donnée, etc.), la méthode utilisée dans la décomposition de salaire (Oaxaca (1973), Oaxaca et Ransom (1994), Neumann et Oaxaca (2004), etc.), la nature et la multiplicité des variables de contrôle, et le choix de la variable salaire (horaire, mensuel, annuel). Néanmoins, elles ont montré, toutes, l'existence d'un supplément de salaire non justifié accordé aux hommes. Nous citons, sans être exhaustifs, certains de ces travaux. Par exemple, Wood, Corcoran et Courant (1993) ont centré leur travail sur un groupe homogène de diplômés de l'école de droit de l'Université de Michigan pendant la période 1972 - I975. L'étude a révélé que l'écart du salaire entre les femmes et les hommes de l'échantillon est moins important au début de carrière. En effet, après quinze ans de vie professionnelle, les femmes reçoivent des salaires $40 \%$ moins importants que ceux des hommes. Ransom et Megdal (1993) se sont interrogés sur l'écart des salaires entre les femmes et les hommes dans le milieu académique, ils ont déduit que malgré l'accroissement de la rémunération féminine, dans le milieu universitaire, depuis les années I960, celle des hommes demeure plus importante. De même Bertrand et Hallock (200I) et Gander (1997) ont mis en évidence la discrimination dans le milieu universitaire. En France, Bayet (1996) a soulevé le problème de discrimination salariale contre les françaises. Il a conclu un écart de salaire non justifié de l'ordre de I4\%. Par ailleurs, l'étude faite par Meurs et Ponthieu (2000) a révélé un écart total en faveur des hommes atteignant les $27 \%$. Cependant, à durée de travail hebdomadaire égale, l'écart est réduit à $16 \%$.

De notre côté, nous allons nous intéresser, dans la présente étude, aux équations de salaires des hommes et des femmes au Maroc. Nous déterminons, ensuite, l'écart salarial entre les deux genres ainsi que les facteurs qui expliquent cette différence de salaire. Nous dégageons, enfin, la part non expliquée de l'écart salarial, pour mettre en évidence le problème de discrimination salariale. Notre étude s'étalera sur les années I990 tout en espérant l'enrichir par l'analyse de données plus récentes afin de couvrir les deux dernières décennies.

Pour ce faire, notre papier comporte cinq parties. Dans la première, nous présenterons le cadre général de la situation des femmes au Maroc. Ensuite, nous expliciterons la méthodologie suivie pour l'élaboration économétrique du problème de la discrimination. Nous consacrerons la troisième partie à la présentation de la base de données utilisée. La quatrième partie mettra en évidence les résultats de nos estimations et, enfin, nous concluons. 


\section{Cadre général}

Au niveau du contexte social, nous avons pu distinguer deux types d'idéologies. D’une part, l'idéologie islamique qui a participé à la reproduction d'une construction socioculturelle des relations entre les genres masculin et féminin en vertu de laquelle, l'image dominante de la femme reste celle d'une "mère et épouse" vouée exclusivement aux tâches ménagères et maternelles. D'autre part, l'idéologie de la famille qui considère que tous les individus existent en tant qu'êtres sexués (hommes ou femmes) et sont socialisés en fonction d'un cadre idéologique associant "la supériorité" aux premiers et "linfériorité" aux secondes.

Sur le plan législatif, et en vertu du principe "A travail égal, salaire égal", la discrimination entre hommes et femmes au Maroc a été interdite (Ratification des Conventions Internationales du Travail, de la convention $C E D A W$, ...). D'autant plus que d'après le nouveau Code du Travail: la maternité a été protégée de telle sorte que les femmes doivent être obligatoirement dispensées de certains travaux pénibles et dangereux, l'exercice des travaux nocturnes doit satisfaire des conditions spécifiques.

Par ailleurs, et au niveau politique, la position de la femme a connu plusieurs avancées au fil des cinquante dernières années. En effet, en 1963, le Maroc a connu ses premières élections législatives d'après l'indépendance. En I976, ily a eu la ratification de la Convention internationale sur les droits politiques de la femme, et en I979, la ratification du Pacte international relatif aux droits civils et politiques (CCPR). En revanche, ce n'est qu'en I993 que la première femme marocaine ait siégé au Parlement dont les députées femmes représentaient $0,9 \%$ pour passer à Io,8\% en 2002. Ceci étant, les femmes au Gouvernement sont passées de $9,75 \%$ en 1995 à $8 \%$ en 2002. Ce recul dans la représentation féminine au Gouvernement ne reflète guère une détérioration de la position de la femme dans le Gouvernement. Bien au contraire, les développements qui vont suivre amorceront l'idée d'une amélioration incessante de la place qu'occupe la femme marocaine au sein de sa société.

S'agissant de la participation économique de la femme au Maroc, il s'est avéré qu'au milieu des années 1970, le pays avait adopté un modèle de développement concentré sur les industries intensives en travail et orienté vers les activités exportatrices telles que le textile. Durant les années I980, la récession économique qu'a connue la région a mis en question la participation féminine qui a engendré un recul sur le marché du travail. Et ce n'est qu'au fil des années I990, que les développements ayant eu lieu avaient mis en exergue le profil genre et avaient contribué à en accroître l'importance pour qu'il occupe une place centrale dans l'élaboration des politiques économiques. 
A partir de 2000 , la participation féminine sur le marché du travail marocain ne cessait de s'accroitre pour être placée au premier rang comparée aux autres pays de la Région MENA. En effet, la figure i montre qu'en 2008 , le taux d'activité féminine avoisinait les $27 \%$ alors que celui des hommes avait atteint les $76 \%$. Cependant, pour les autres pays arabes, le taux d'activité féminine était de l'ordre de $26 \%$ pour la Tunisie, $23 \%$ pour l'Egypte, $2 \mathrm{r} \%$ pour le Liban, etc. S'agissant des taux d'activité masculine pour ces pays, ceux-ci fluctuaient entre $72 \%$ pour l'Egypte, $68 \%$ pour la Tunisie et $66 \%$ pour le Liban.

Par ailleurs, les taux de participation féminine et masculine dans les pays de l'OCDE sont un peu plus élevés pour les hommes mais beaucoup plus élevés pour les femmes. En fait, les femmes des pays développés ont un taux de participation au marché du travail de l'ordre de $74 \%$ pour le Canada, $70 \%$ pour l'Allemagne, $65 \%$ pour la France, etc. Les taux d'activité masculine dépassent ceux des femmes de peu. Faudrait-il remarquer, cependant, que le taux de chômage féminin dans les pays arabes pourrait atteindre le triple de celui des pays de l'OCDE. Tandis que celui des hommes est presque le double (Figure I).

Il est clair donc, que les femmes dans nos pays arabes participent moins sur le marché du travail que celles des pays développés. Ceci peut être dû à une évolution tardive et un contexte sociopolitique et culturel pas suffisamment développés pour permettre à la femme marocaine en particulier et arabe en général de s'infiltrer dans la vie économique de façon beaucoup plus opérationnelle et productive.

Figure1 : Taux de participation au marché du travail et taux de chômage par pays (zones MENA) et sexes

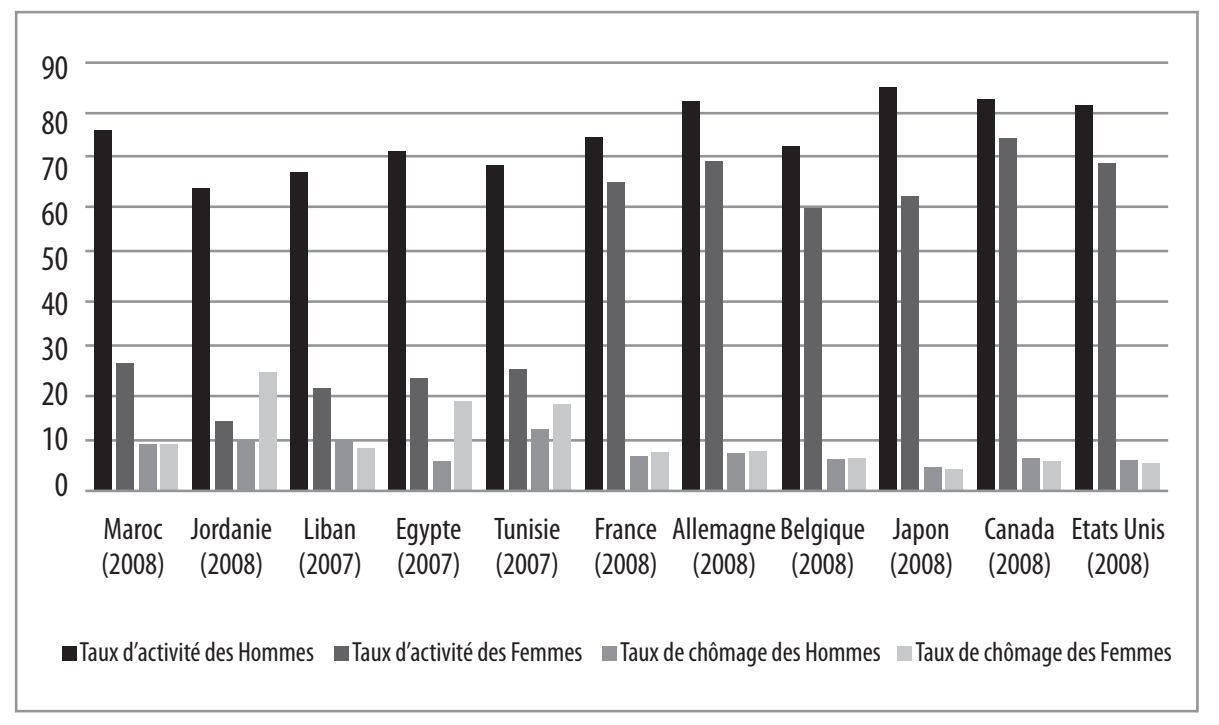


Afin de diagnostiquer la situation au Maroc de façon plus désagrégée, il importe de s'interroger sur l'évolution des taux d'activité par genre et groupe d'âge en un premier temps, pour mettre en lumière la dispersion du taux de chômage par genre et groupe d'âge en un deuxième temps. En effet, la figure 2 montre que la participation la plus importante enregistrée, aussi bien pour les hommes que pour les femmes, touche les tranches d'âge compris entre 25 et 44 ans. Le niveau le plus bas était enregistré pour les deux sexes en début de leur carrière ( $15-24$ ans). De même, les taux d'activités commencent leur chute à partir de 44 ans pour atteindre $69 \%$ pour les hommes et $24 \%$ pour les femmes en fin de carrière.

Figure 2: Taux d'activité des marocains par sexes et âge, 2008

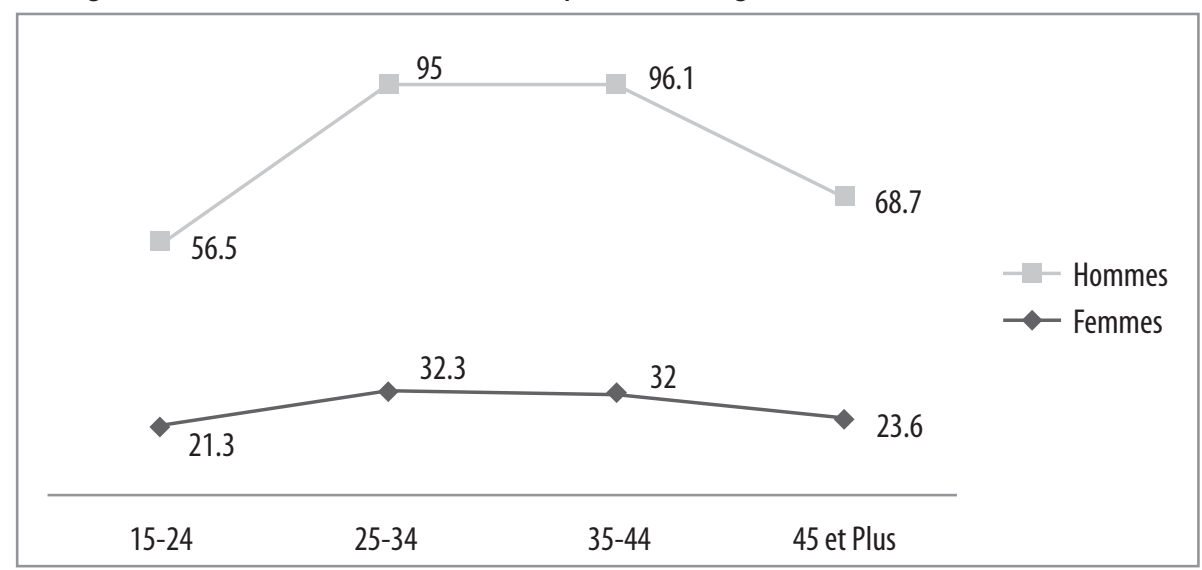

Ainsi, la conclusion qui peut être faite à ce niveau c'est que, comme partout dans les pays de la région MENA, les femmes marocaines ont une faible participation sur le marché du travail et ce même durant l'intervalle d'âge où l'individu est censé être le plus actif. Sinon, pour ce qui est de l'évolution de la participation durant le cycle de vie, il était tout à fait comparable pour les deux sexes traduisant ainsi la subdivision de la vie active en trois étapes importantes: la préparation à la vie professionnelle où l'entrée se fait de façon progressive ; la pleine activité ; et, l'achèvement graduel de la vie active.

Par ailleurs, la figure 3 traduit l'évolution des taux de chômage par genre et groupe d'âge durant la période $2000-2008$. Il est évident de constater que les taux de chômage les plus élevés s'étalent sur l'intervalle d'âge 15 - 34. En effet, les jeunes qui viennent de débarquer sur le marché du travail sont les plus susceptibles de se trouver au chômage vu leur manque d'expérience. A partir de 35 ans, le taux de chômage commence à se replier aussi bien pour les hommes que pour les femmes pour atteindre ses niveaux les plus bas à partir des 45 ans. S'agissant de l'évolution des taux de chômage masculin et féminin entre 2000 et 2008 , la figure 3 traduit 
une faible baisse en 2008. En totalité, la baisse avait été plutôt favorable à la main d'œuvre masculine dont le taux de chômage est passé de 13 à $9 \%$. Cependant, celui des femmes n'avait bougé que de 3 points en passant de I $2,5 \%$ à $9,5 \%$.

Figure 3 :Taux de cômage marocain par sexe et âge

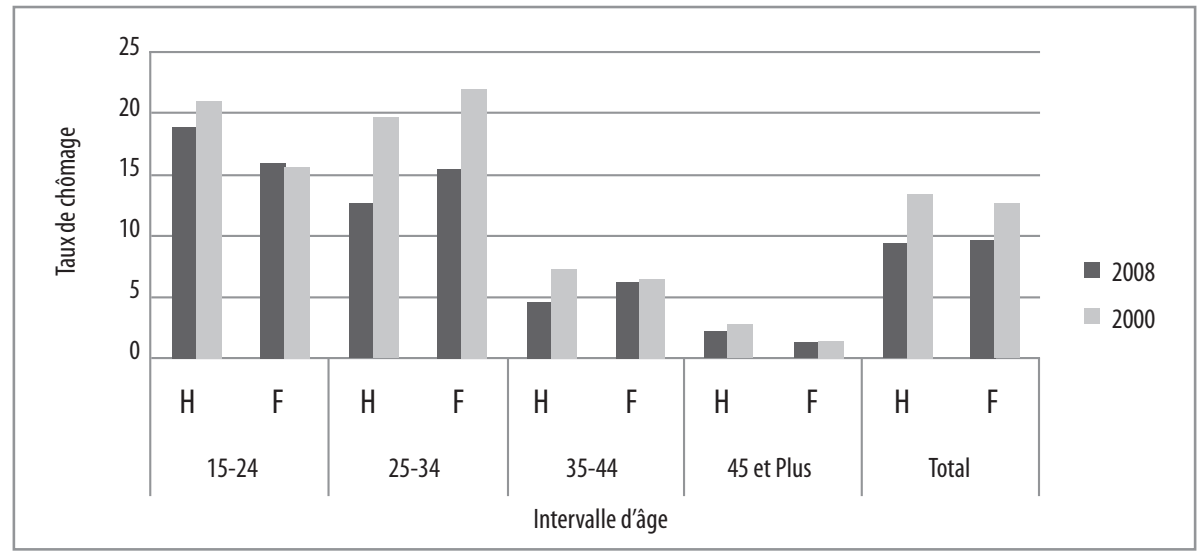

Ainsi, et en tenant compte de l'analyse descriptive qui a précédé, il importe de s'interroger dans ce qui suit sur l'ampleur de la discrimination rencontrée par les marocaines lors de l'embauche. Nous traiterons particulièrement la discrimination salariale en utilisant les méthodes de décompositions qui seront décrites dans le paragraphe suivant.

\section{Méthodologie}

La plupart des études économétriques s'inspirent des travaux de Oaxaca (I973) et de Blinder (1973). La méthode consiste à estimer séparément l'équation de salaire des hommes et des femmes, puis calculer l'écart de salaire entre les deux sexes au niveau moyen :

Soit:

$$
\begin{aligned}
& w_{i h}=x_{h} \beta_{h}+\varepsilon_{h} \\
& w_{i f}=x_{f} \beta_{f}+\varepsilon_{f}
\end{aligned}
$$

Où $\mathrm{w}_{\text {if }}$ et $\mathrm{w}_{\text {ih }}$ sont respectivement les logarithmes des salaires des femmes et des hommes. $\mathrm{x}_{\text {if }}$ et $x_{i h}$ l'ensemble des variables explicatives qui déterminent le salaire. Elles incluent les caractéristiques du capital humain telles que l'expérience, l'ancienneté, le diplôme, etc. Aussi, les caractéristiques de l'emploi telles que: les catégories socioprofessionnelles, 
le type de contrat de travail, ... et, enfin, les caractéristiques de l'entreprise comme la taille, le secteur, etc.

Lécart de salaire moyen entre les hommes et les femmes est alors:

$$
\bar{w}_{h}-\bar{w}_{f}=\hat{\beta}_{h}\left(\bar{x}_{h}-\bar{x}_{f}\right)+\bar{x}_{f}\left(\hat{\beta}_{h}-\hat{\beta}_{f}\right)
$$

Le premier terme de l'écart $\left(\hat{\beta}_{h}\left(\bar{x}_{h}-\bar{x}_{f}\right)\right)$ est la part due à une différence de caractéristiques moyennes entre les hommes et les femmes. Elle serait donc la part expliquée de l'écart liée à une différence de productivité entre les deux groupes. Cette composante ${ }^{\mathrm{I}}$ serait nulle si les deux groupes avaient les mêmes caractéristiques. Le second terme $\left(\bar{x}_{f}\left(\hat{\beta}_{h}-\hat{\beta}_{f}\right)\right)$, est la part de l'écart due à une différence de rémunération de ces caractéristiques: c'est la part inexpliquée. Cette composante est assimilée à la discrimination salariale ${ }^{2}$. En absence de toute discrimination $\left(\hat{\beta}_{h}=\hat{\beta}_{f}\right)$, elle serait nulle.

Cette décomposition propose comme référence de rémunération non discriminante celle du groupe dominant c'est-à-dire celle des hommes ( $\hat{\beta}_{h}$ ). Ce choix a été souvent critiqué. En effet, le choix de la référence non discriminante affecte les résultats, notamment, la composante de l'écart salarial assimilée à la discrimination. Il serait donc hardi de supposer d'emblée que les hommes ne sont pas discriminés pour prouver l'existence de la discrimination. Oaxaca et Ransom (1994) proposent une nouvelle décomposition dans laquelle la référence de rémunération des caractéristiques non discriminante est $\left(\beta^{*}\right)$ :

$$
\bar{w}_{h}-\bar{w}_{f}=\underbrace{\beta^{*}\left(\bar{x}_{h}-\bar{x}_{f}\right)}_{1}+\underbrace{\bar{x}_{h}\left(\hat{\beta}_{h}-\beta^{*}\right)}_{2}+\underbrace{\bar{x}_{f}\left(\beta^{*}-\hat{\beta}_{f}\right)}_{3}
$$

Ils suggèrent d'estimer $\left(\beta^{*}\right)$ à partir de l'équation de gain de l'ensemble de l'échantillon contenant les hommes et les femmes.

Lidée de cette nouvelle décomposition est que la discrimination peut être non seulement au détriment des femmes mais aussi en faveur des hommes. Le premier terme de cette décomposition est l'écart de salaire dû à une différence de caractéristiques entre les deux genres. Le second terme est le supplément de rémunération des caractéristiques accordé

1 Cette composante reflète la partie non discriminante du salaire si les caractéristiques reflètent les véritables choix et les véritables productivités des travailleurs. Or les postes occupés par les femmes peuvent être affectés par des pratiques discriminatoires soit à l'embauche, soit à l'accès aux promotions.

2 Cette décomposition est sensible au choix de variables, (Proxy de l'expérience professionnelle), sensible également aux coefficients estimés (problème de sélection, etc.) 
aux hommes par rapport à la norme: c'est l'avantage masculin. Le dernier terme est la différence de rémunération des caractéristiques des femmes par rapport à la norme : c'est le désavantage féminin.

Pour résumer, le premier terme est la part justifiée ou expliquée de l'écart salarial. Le second et le troisième termes forment la composante non expliquée de l'écart salarial: c'est l'écart salarial dû à une discrimination.

\section{Les données}

Nous nous sommes basés dans cette étude sur l'Enquête Nationale sur les Niveaux de Vie des Ménages qui a eu lieu au Maroc en I990-9I et I99899. Le tableau ci-dessous explicite le contenu de chacune des deux bases de données ainsi que des échantillons utilisés dans la présente étude.

Comme le montre le tableau I, en I99I, 3349 ménages contenant I9677 individus ont été interviewés. Ce nombre était passé à 30457 individus contenus dans 5129 ménages en I999. Le pourcentage des citadins était moins important en I99I qu'en I999 puisqu'il était passé de $66 \%$ à $74 \%$. Par ailleurs, afin d'accomplir la présente recherche, nous nous sommes basés sur deux échantillons contenant 1944 individus, dont 1563 hommes, extraits de l'enquête I99I ; et 3847 individus, dont 2925 hommes, extraits de celle de 1999 .

Tableau 1: Description des échantillons

\begin{tabular}{|l|c|c|}
\hline $\begin{array}{l}\text { Enquête Nationale sur les } \\
\text { Niveaux de Vie des Ménages }\end{array}$ & $1990 / 91$ & $1998 / 99$ \\
\hline Echantillon total: & 19677 & 30457 \\
Individus & 3349 & 5129 \\
Ménages & $66 \%$ & $74 \%$ \\
\hline Citadins & 1944 & 3847 \\
\hline Echantillon sélectionné \\
(effectif des individus)
\end{tabular}

Les tableaux 2 et 3 expriment les statistiques descriptives des variables utilisées dans les équations de salaires de I99I et I999 qui seront estimées ultérieurement. La première remarque qu'il faudrait mettre en évidence est l'augmentation du salaire hebdomadaire entre les deux périodes analysées. En effet, le salaire hebdomadaire moyen est passé de 380 Dirhams (DH) en I99I à $434 \mathrm{DH}$ en 1999 réalisant ainsi une augmentation de I4\%. Cette augmentation avait été sensiblement supérieure pour les hommes (I6\% 
contre $13,4 \%$ ). Cependant, du côté des secteurs privé et public, il a été démontré que l'augmentation enregistrée dans le secteur public est plus importante que celle du secteur privé. En effet, alors que dans ce dernier, le salaire hebdomadaire avait augmenté de 21,5\%, l'accroissement dans le secteur public était de l'ordre de $26 \%$. Ce pourcentage élevé cache une augmentation très importante au profit des femmes travaillant dans ce secteur ( $36,8 \%$ contre $25 \%$ pour les hommes). Toutefois, dans le secteur privé, une faible évolution du salaire hebdomadaire féminin ( $13 \%$ ) a été confrontée à une augmentation plutôt importante des salaires des hommes $(25 \%)$. Ainsi, il est clair qu'au sein du secteur privé, l'accroissement du salaire féminin n'était guère suffisant pour combler la différence de salaire entre les hommes et les femmes (les hommes gagnent $25 \%$ de plus que les femmes en I991 et $38 \%$ de plus en 1999). Dans le secteur public, la situation bien qu'elle s'est améliorée entre I99I et 1999 mais n'était pas tout-àfait meilleure puisque en I99I les hommes gagnent $36 \%$ de plus que les femmes. Cette différence chute et atteint $24 \%$ en 1999 . Par ailleurs, il a été constaté que les hommes sont rémunérés beaucoup mieux dans le public que dans le privé (un peu plus que le double) alors que la rémunération féminine dans le public est $88 \%$ plus importante que dans le privé pour ce qui est de 1991. En 1999, la situation n'avait presque pas bougé pour les hommes. Cependant, pour les femmes, l'amélioration était nette puisque celles-ci ont vu leur salaire hebdomadaire plus que doublé dans le public comparé au privé (le salaire hebdomadaire dans le public est égal à $227 \%$ de celui du privé).

Ces améliorations ne sont guère dues au hasard. En effet, les efforts dans l'éducation ont été le principal facteur dans l'accroissement des salaires hebdomadaires pour les hommes et les femmes au sein des secteurs chacun à part et entre les deux périodes étudiées. D'après les tableaux 2 et 3, le pourcentage des illettrés était de l'ordre de 39 et $44 \%$ successivement pour les hommes et les femmes en I99I ; ces taux ont légèrement baissé en I999 et ont passé à 35 et 39\%. D'autant plus que la distribution des niveaux éducatifs a été modifié et nous avons assisté plutôt à une tendance vers la technicité de l'éducation. En effet, aussi bien pour les hommes que pour les femmes, l'enseignement technique a pris de l'élan par rapport aux autres niveaux enregistrant ainsi un accroissement remarquable (ceux ayant un diplôme technique sont devenus 2,33 fois plus dans le public et exactement le double dans le privé et ce en passant de 1991 à 1999). Ensuite le niveau universitaire a enregistré une montée assez importante dans le privé puisque l'effectif de ceux ayant un niveau universitaire et travaillant dans le privé était passé du simple au double entre 1991 et 1999. Les universitaires travaillant dans le secteur public n'ont pas beaucoup bougé entre les deux périodes mis à part la hausse négligeable de l'effectif des femmes ayant un niveau universitaire et travaillant dans ce secteur (28\% de plus en 1999). Lautre élément intéressant est que si on compare la distribution des niveaux 
éducatifs entre le public et le privé, nous remarquerons que le secteur public accapare pour lui-même 15 fois plus de diplômés de l'université marocaine en I991. Cette proportion est passée à 7 fois en 1999 pour ainsi confirmer une remarque précédente selon laquelle l'effectif universitaire dans le privé avait atteint le double entre i991 et 1999. S'agissant des autres niveaux éducatifs, les tendances ont été nuancées entre hausse et baisse selon les périodes étudiées, les secteurs et les deux sexes. Mais l'élément marquant est que ceux et celles ayant un niveau secondaire deuxième cycle se sont vus leurs parts du marché du travail chuter considérablement. Ceci reflète la conscience accrue des responsables dans les secteurs public et privé quant à la nécessité d'embaucher des gens qui ont une qualification minimale acquise soit à l'université soit à l'enseignement technique, sinon, des gens carrément non qualifiés.

Pour ce qui est de l'expérience, il s'avère qu'entre I99I et 1999, les choses n'ont pas considérablement évolué. En effet, la moyenne du nombre d'années d'expérience est restée presque la même aussi bien pour les hommes que pour les femmes, et pareillement dans le public et le privé. Cependant, en I99I, il apparaît que les hommes du public ont $25 \%$ plus d'expérience que les femmes du même secteur. Dans le privé, le décalage n'est que de $8 \%$ toujours en faveur du sexe masculin. En revanche, en I999, la situation s'était inversée pour donner au secteur privé l'avantage par rapport au public. Les hommes travaillant dans ce dernier ont en moyenne II \% plus d'expérience que les femmes. Ce taux grimpera à I $8 \%$ dans le secteur privé. Il s'avère évident, qu'au sein du privé l'écart se creuse de plus en plus alors que dans le public, cet écart s'est plutôt replié.

Par ailleurs, l'emploi dans le secteur public a baissé entre i99i et 1999 de presque $25 \%$ dans l'ensemble avec une légère avancée pour les femmes (30\% contre $26 \%$ pour les hommes). Cependant, le rapport homme/femme dans l'emploi public était resté presque égal à l'unité pour chacune des deux années étudiées pour ainsi révéler une parité quasi-identique pour les deux sexes dans ce secteur.

Ainsi, l'analyse descriptive précédente révèle bel et bien une évolution, dans sa globalité positive, de la participation des femmes sur le marché du travail au Maroc. Cette évolution n'aurait pu être concrétisée sans les avancées enregistrées essentiellement sur le plan éducatif où les femmes ont pu se partager les bancs de l'école, du lycée et de l'université avec leurs homologues masculins. Ceci n'empêche pas d'évoquer l'importance de l'expérience qui a pu, elle aussi, améliorer le sort des femmes sur le marché du travail dans le pays aussi bien dans les secteurs public et privé. Dans ce qui suit, nous essayerons de rentrer davantage dans le cœur de l'analyse en s'interrogeant sur l'ampleur et les déterminants des salaires des hommes et des femmes dans les deux secteurs public et privé pour aboutir à une décomposition explicite de l'écart de salaire entre les deux sexes tout en essayant d'en détecter les origines. 


\begin{tabular}{|c|c|c|c|c|c|c|c|c|c|c|}
\hline & 焉 & 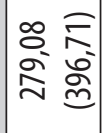 & 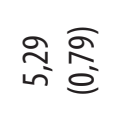 & 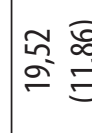 & 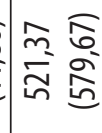 & 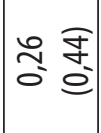 & $\therefore \overline{\widetilde{m}}$ & 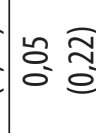 & $\stackrel{0}{0}$ & $\delta_{0}$ \\
\hline 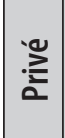 & 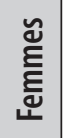 & 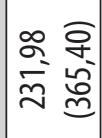 & 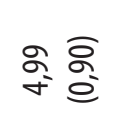 & 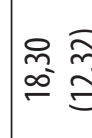 & 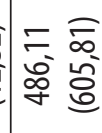 & & $\stackrel{\widetilde{\Xi}}{\stackrel{\Xi}{\Xi}}$ & : & ì & $\stackrel{\frac{m}{s}}{O}$ \\
\hline & 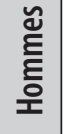 & 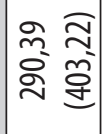 & 总 & $\begin{array}{l}\bar{\sigma} \\
\bar{\sigma} \\
\sigma\end{array}$ & 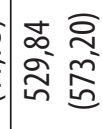 & & 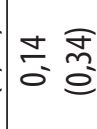 & & & $\bar{\sigma}$ \\
\hline & 慇 & 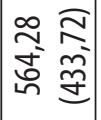 & 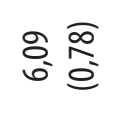 & $\begin{array}{l}\hat{\sigma} \\
\approx \bar{z}\end{array}$ & $\begin{array}{l}\alpha \\
\alpha \\
\alpha\end{array}$ & & $\infty^{\infty}$ & $\frac{\infty}{0} \stackrel{\Omega}{\Omega}$ & 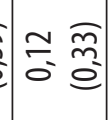 & a \\
\hline 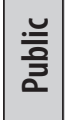 & 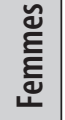 & 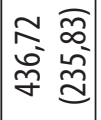 & 㣽 & 茫 & 总 & 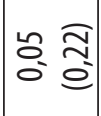 & $\frac{\pi}{0}$ & $\bar{\sigma}$ & 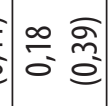 & 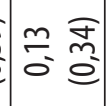 \\
\hline & 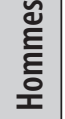 & 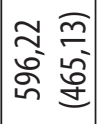 & $\begin{array}{l}\frac{n}{2} \frac{F}{b} \\
\frac{0}{0}\end{array}$ & 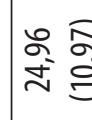 & 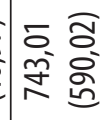 & 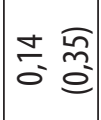 & 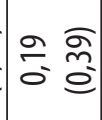 & $\frac{\infty}{\infty}$ & $=\frac{\bar{m}}{\varrho}$ & 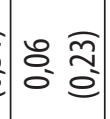 \\
\hline 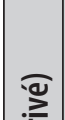 & 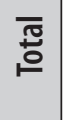 & 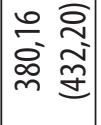 & 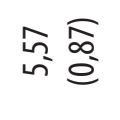 & 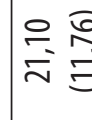 & 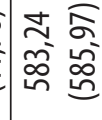 & 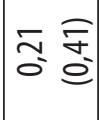 & 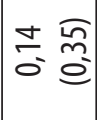 & 응 & $\stackrel{8}{\stackrel{8}{s}}$ & $\frac{\infty}{0}$ \\
\hline 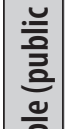 & 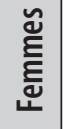 & 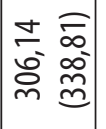 & 总 & $\begin{array}{ll}\infty & \sigma \\
\infty & \vdots \\
\infty & =\end{array}$ & 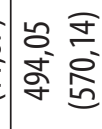 & 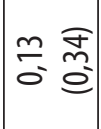 & $\begin{array}{lll}8 & \bar{\Xi} \\
0 & 0 & 0\end{array}$ & 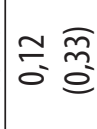 & {$\left[\begin{array}{ll}0 & \overline{2} \\
0 & 0 \\
0\end{array}\right.$} & 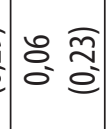 \\
\hline 푸 & 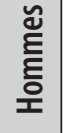 & 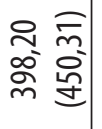 & $\begin{array}{l}\dot{t} \widetilde{\infty} \\
\text { in }\end{array}$ & 兑 & {$\left[\begin{array}{ll}\infty & \\
0 \\
0 \\
0\end{array}\right.$} & $\begin{array}{lll}\approx & \widetilde{f} \\
0 & =\end{array}$ & 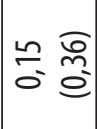 & o & 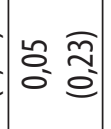 & $\stackrel{0}{0}$ \\
\hline & & 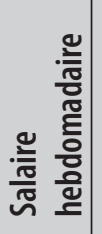 & 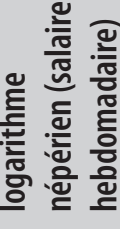 & 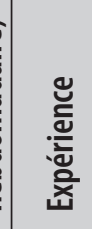 & 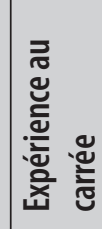 & 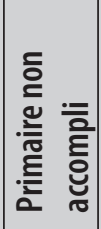 & $\begin{array}{l}\text { 絯 } \\
\text { 产 }\end{array}$ & 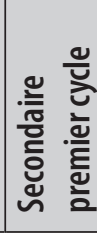 & 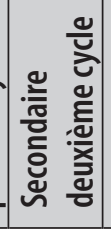 & 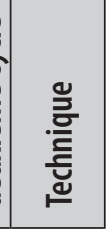 \\
\hline
\end{tabular}




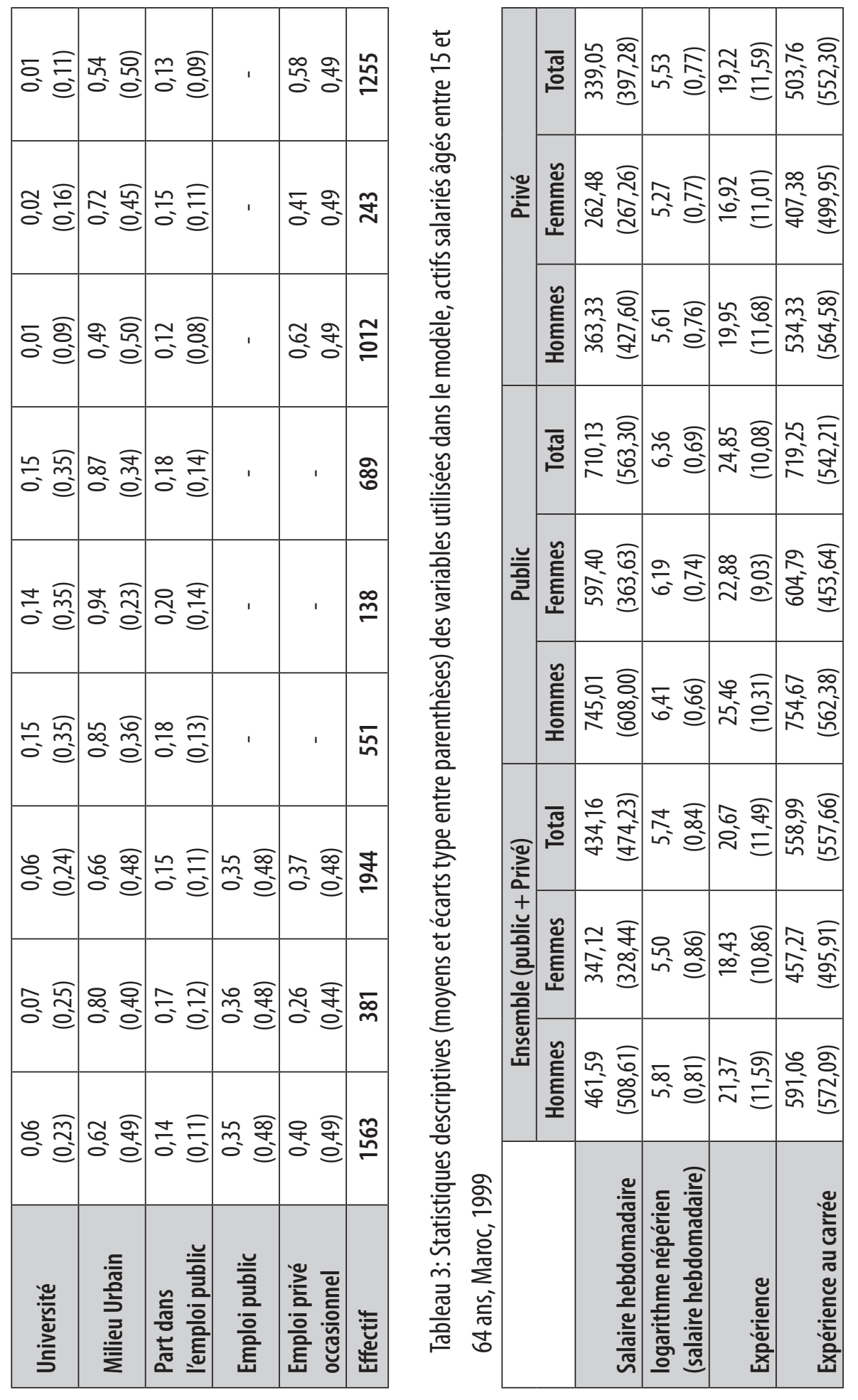




\begin{tabular}{|c|c|c|c|c|c|c|c|c|c|c|c|}
\hline 必 & 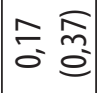 & 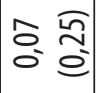 & $\begin{array}{ll}0 & \overline{0} \\
0 & 0 \\
0\end{array}$ & $\approx \frac{\pi}{0}$ & $\begin{array}{l}\approx \\
0 \\
0\end{array}$ & $\mid \begin{array}{ll}5 \\
0\end{array}$ & 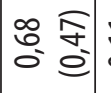 & 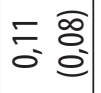 & & & i d \\
\hline $\begin{array}{l}0 \\
\\
\end{array}$ & 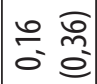 & $\begin{array}{ll}0 & \bar{\Omega} \\
0 & 0 \\
0 & 0\end{array}$ & 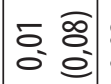 & $\begin{array}{l}2 \\
0 \\
0\end{array}$ & $\tilde{O}: \frac{\sigma}{\sigma}$ & $\mid \begin{array}{cc}0 & \overline{0} \\
0 & 0 \\
0\end{array}$ & 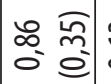 & $\begin{array}{ll}m & \bar{o} \\
0 & 0 \\
& 0\end{array}$ & & $\bar{a}=0$ & : \\
\hline 商亭 & $\left|\begin{array}{cc}1 & \widehat{\alpha} \\
0 & 0 \\
0 & =\end{array}\right|$ & & $\mid$ & $\frac{\approx}{0} \frac{\pi}{0}$ & $\frac{\bar{\delta}}{\overline{0}} \overline{\bar{e}}$ & $\left(\begin{array}{cc}0 \\
0 \\
0\end{array}\right.$ & 空 & $\begin{array}{ll}0 \\
0 \\
0\end{array}$ & & & $\stackrel{\Sigma}{\beth}$ \\
\hline & 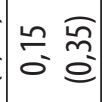 & 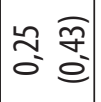 & 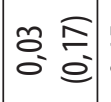 & 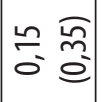 & 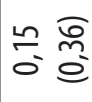 & $\left(\begin{array}{cc}0 \\
0\end{array}\right.$ & 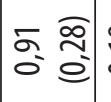 & $\begin{array}{ll}\infty & 0 \\
\frac{0}{0} & \frac{0}{6}\end{array}$ & & & œ \\
\hline & 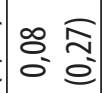 & $\begin{array}{lll}\tilde{\sigma} & & \bar{f} \\
\tilde{\sigma} & 0\end{array}$ & 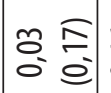 & $\frac{0}{0} \stackrel{\widetilde{m}}{0}$ & $\frac{\infty}{\sigma} \bar{\alpha} \frac{\bar{\alpha}}{e}$ & $\begin{array}{cc}0 & \overline{0} \\
0 & 0\end{array}$ & $\mid \begin{array}{ccc}\infty & \frac{n}{0} \\
0 & 0 & 0\end{array}$ & $\begin{array}{lll}0 & 0 \\
0 & 0 \\
0 & 0\end{array}$ & & & $\tilde{\approx}$ \\
\hline 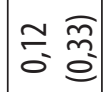 & 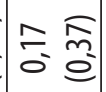 & 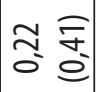 & $\mid \begin{array}{cc}0 \\
0 \\
0\end{array}$ & 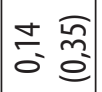 & 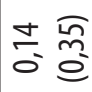 & $\left(\begin{array}{cc}\overline{0} \\
0\end{array}\right.$ & {$\left[\begin{array}{cc}a \\
0 \\
0\end{array}\right.$} & $\frac{\infty}{0} \stackrel{\frac{\pi}{5}}{=}$ & & & $\Re$ \\
\hline & 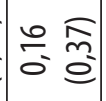 & 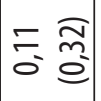 & $\left(\begin{array}{ll}\bar{E} \\
0\end{array}\right.$ & 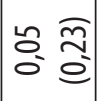 & 녕 & $\begin{array}{lll}\bar{\theta} & \\
0 & 0\end{array}$ & 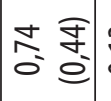 & $\begin{array}{lll}m & \\
0 & = \\
0 & =\end{array}$ & $\begin{array}{l}\stackrel{F}{f} \\
0\end{array}$ & & 唐 \\
\hline & 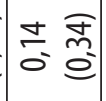 & 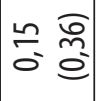 & {$\left[\begin{array}{cc}\bar{E} \\
0 \\
0\end{array}\right.$} & $\begin{array}{lll}8 & \bar{\Xi} \\
0 & 0\end{array}$ & 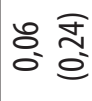 & $\begin{array}{cc}z & \bar{m} \\
0 & 0\end{array}$ & $\mid \begin{array}{ccc}\infty & \bar{p} \\
0 & \stackrel{0}{0}\end{array}$ & 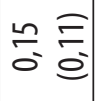 & $\begin{array}{ll}\stackrel{a}{\sigma} \\
\delta \\
\sigma\end{array}$ & & $\approx$ \\
\hline 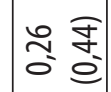 & 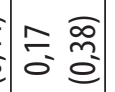 & $\mid \begin{array}{cc}0 \\
\frac{0}{0} & 0 \\
0\end{array}$ & $\left(\begin{array}{cc}\overline{0} \\
0\end{array}\right.$ & $\begin{array}{ll}2 & \overline{\mathbb{z}} \\
0 & \underline{\Xi}\end{array}$ & 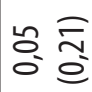 & $\mid \begin{array}{cc}5 & = \\
0 & 0 \\
0\end{array}$ & $\mid \begin{array}{cc}0 & 0 \\
0 & 0 \\
0 & 0\end{array}$ & $\begin{array}{lll} & & = \\
0 & 0 & 0\end{array}$ & 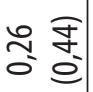 & & $\stackrel{2}{2}$ \\
\hline 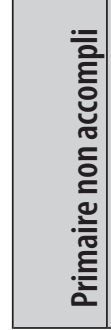 & $\mid$ & 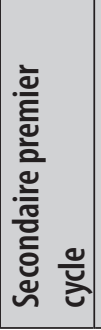 & 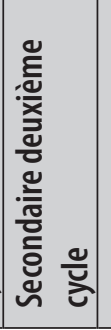 & 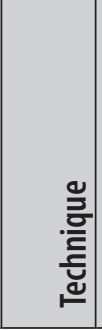 & 总 & 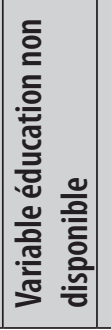 & 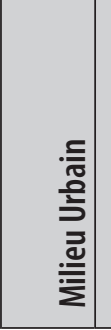 & 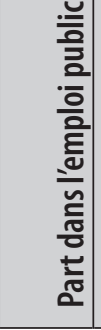 & 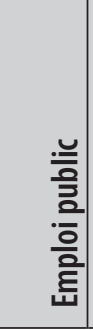 & 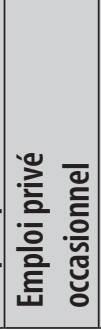 & $\begin{array}{l}\text { 壳 } \\
\text { 密 }\end{array}$ \\
\hline
\end{tabular}




\section{Estimations}

Avant de mettre en évidence les résultats de notre travail empirique, il importe de signaler le handicape rencontré lors des estimations à savoir l'absence d'information sur le nombre d'heures travaillées par les salariés en 1999. En effet, il aurait été d'un grand intérêt de réaliser nos estimations en nous basant sur les salaires horaires des travailleurs afin de converger vers la théorie du capital humain. Hélas, pour ce qui est de 1999, les données disponibles, malgré leurs pertinences et richesses, n'incluent pas d'informations sur le nombre d'heures travaillées ce qui a empêché tout travail empirique conforme à la théorie. Comme tentative pour surmonter ce problème et pour que notre analyse soit la plus plausible et fiable possible, nous avons essayé de nous baser sur les salaires hebdomadaires pour les deux périodes.

S'agissant des résultats des estimations, ceux-ci ont révélé l'importance de l'éducation comme élément prépondérant dans la formation de salaire (Tableaux 4 et 5). D'ailleurs, cette importance s'est manifestement concrétisée à travers les coefficients des variables explicatives utilisées dans chaque modèle. En effet, le rendement de l'éducation augmente au fur et à mesure que l'on avance dans les niveaux éducatifs. C'est dans ce sens que le rendement du primaire est 5 fois moins important que le niveau d'éducation universitaire dans le cas des hommes marocains en I99I. En I999, la progression du même taux était beaucoup plus intense puisque le niveau d'éducation primaire des hommes travaillant en tant que salariés contribue à raison de 0,188 point seulement comparé à la valeur ajoutée d'un diplôme universitaire qui avoisinée les I,29 points. Ceci n'est qu'un exemple parmi d'autres.

Lautre constat, aussi important que le premier, c'est la différence dans les rémunérations entre le privé et le public. D’ailleurs, El-Hamidi et Said (2005) ont évoqué cette différence de rémunération pour le même sexe. Notre étude avait tout de même détecté cette différence sans rentrer dans ses détails puisque l'objet de la présente recherche est de calculer les écarts salariaux en fonction du genre et non pas en fonction des secteurs. Pour rester toujours dans l'éducation, il faut remarquer que les femmes ont presque, à tous les niveaux, un rendement supérieur aux hommes. Cependant, et malgré les différences qui existent dans le rendement selon les niveaux éducatifs, c'est dans le secteur public qu'on trouve l'écart le plus important entre le rendement féminin et celui masculin opérant toujours en faveur des femmes. Par ailleurs, un cas nécessite d'être analysé à part, à savoir le rendement de l'université chez les hommes en I99I qui avait dépassé, quoique de peu, celui des femmes. Ceci peut être expliqué 
par le fait que dans le privé, les diplômés de l'université trouvent mieux leur compte que dans le public. Ces remarques et observations seront d'une grande utilité lors de l'interprétation des résultats finaux du calcul des écarts salariaux selon les secteurs.

Par ailleurs, l'expérience contribue significativement à l'accroissement du salaire. Dans les différents cas, hommes ou femmes, privé ou public, le coefficient attribué à cette variable est significativement supérieur à zéro. Ceci confirme, encore une fois, la théorie du capital humain.

Pour ce qui est de l'appartenance au milieu urbain, les tableaux 4 et 5 révèlent qu'habitant dans une zone urbaine pourrait favoriser le salaire hebdomadaire dans le public. En effet, la contribution est surtout apparente dans le cas des femmes où elle a été une fois et demi plus importante en I 999 comparée à I99I (0,983 contre 0,633). De même, l'effet induit dans le cas des hommes travaillant dans ce secteur était aussi presque une fois et demi plus important en 1999 sans pour autant atteindre l'importance des coefficients féminins. De manière générale, être employé dans le secteur public contribue positivement à la rémunération des deux sexes avec une évolution avoisinant les 60\% en allant de I991 à I999. En plus, l'examen de tous les salariés (public et privé) révèle que l'impact d'un emploi dans le public, qui était plus important pour les hommes en 1991, est devenu plus important pour les femmes en 1999.

Par ailleurs, les hommes travaillant dans le secteur privé ont intérêt d'être permanent puisque la variable privé non permanent est significativement négatif pour les hommes en I99I et 1999. Contrairement, il apparaît que les femmes en ont plutôt intérêt puisque ce statut contribue positivement à leur salaire hebdomadaire selon les estimations relatives à l'année I99I. En I999, le coefficient est négatif et non significatif.

S'agissant de l'écart des salaires entre les hommes et les femmes au Maroc, nos estimations ont révélé un écart salarial assez énorme comparé à celui des pays occidentaux (Tableau 6 et Figure 4). En effet, dans l'ensemble, l'écart des salaires entre les hommes et les femmes était de l'ordre de $34,6 \%$ et $30,9 \%$ dans les deux secteurs confondus, et ce successivement pour 1991 et 1999. Par la suite, nous avons essayé de décomposer cet écart entre part justifiée et part non justifiée. Nos calculs ont démontré que la majeure partie de ces écarts est due à des composantes injustifiables. C'est dans ce sens qu'en I99I, 35,7\% de l'écart salarial entre homme et femme est tributaire à la discrimination alors qu'en I999, ce taux était passé à 29,4\%. D'autant plus que l'écart justifié ne représente qu'une portion très réduite ne dépassant pas 1,5\% en 1999 et carrément négative en I99I. Cet écart négatif indique que l'avantage est, dans ce cas là, en faveur des femmes puisque l'écart, mathématiquement parlant, n'est qu'une différence entre deux grandeurs qui peut être soit positif soit négatif. En conclusion, il 
s'avère que, entre 199I et 1999, l'écart salarial avait baissé de presque 4 points. Cette baisse était saisie du côté de l'écart injustifié qui a reculé de 6 points en faveur de l'écart justifié qui était passé de -I,I\% à I, $5 \%$.

Par ailleurs, et dans le but d'élargir notre étude, nous avons décomposé l'écart salarial au sein des secteurs public et privé pris séparément. Cette décomposition avait été d'un grand intérêt puisqu'elle a reflété l'importance de la scolarisation des femmes dans le secteur public. En effet, avec un écart salarial avoisinant $31,5 \%$ en 1991 et $21,8 \%$ en 1999 dans le secteur public, nous avions pu dégager une part assez significative en termes d'écart justifié en faveur des femmes. C'est ainsi, que l'écart justifié qui était égal à $-5,4 \%$ en I99I est passé à -I3,I\% en 1999. Le signe négatif indique un avantage féminin reflétant ainsi l'avancée des femmes en termes d'éducation comme nous l'avons précédemment souligné. Ainsi, au sein du public, et dû à un rendement supérieur de l'éducation chez les femmes, celles-ci possèdent un avantage en termes de rémunération qui essaye d'amortir la discrimination contre elles. D'ailleurs, la part injustifiée de la discrimination salariale dans le public est de l'ordre de $37 \%$ et $35 \%$ successivement en 1991 et 1999. Lesquels taux ont été partiellement anéantis par la part justifiée négative dans les deux cas.

Néanmoins, dans le secteur privé, l'écart salarial était assez important du point de vue valeur. C'est dans ce sens qu'en i99ı, l'écart salarial homme-femme était de l'ordre de $37,5 \%$ pour passer en 1999 à $33,5 \%$. Cette baisse, relativement faible comparée à celle dans le public, bien qu'elle soit accompagnée par une augmentation de l'écart justifié qui est passé de I à $6 \%$, elle a été due essentiellement à la diminution de l'écart injustifié qui est passé de $36,7 \%$ à $27,6 \%$. 


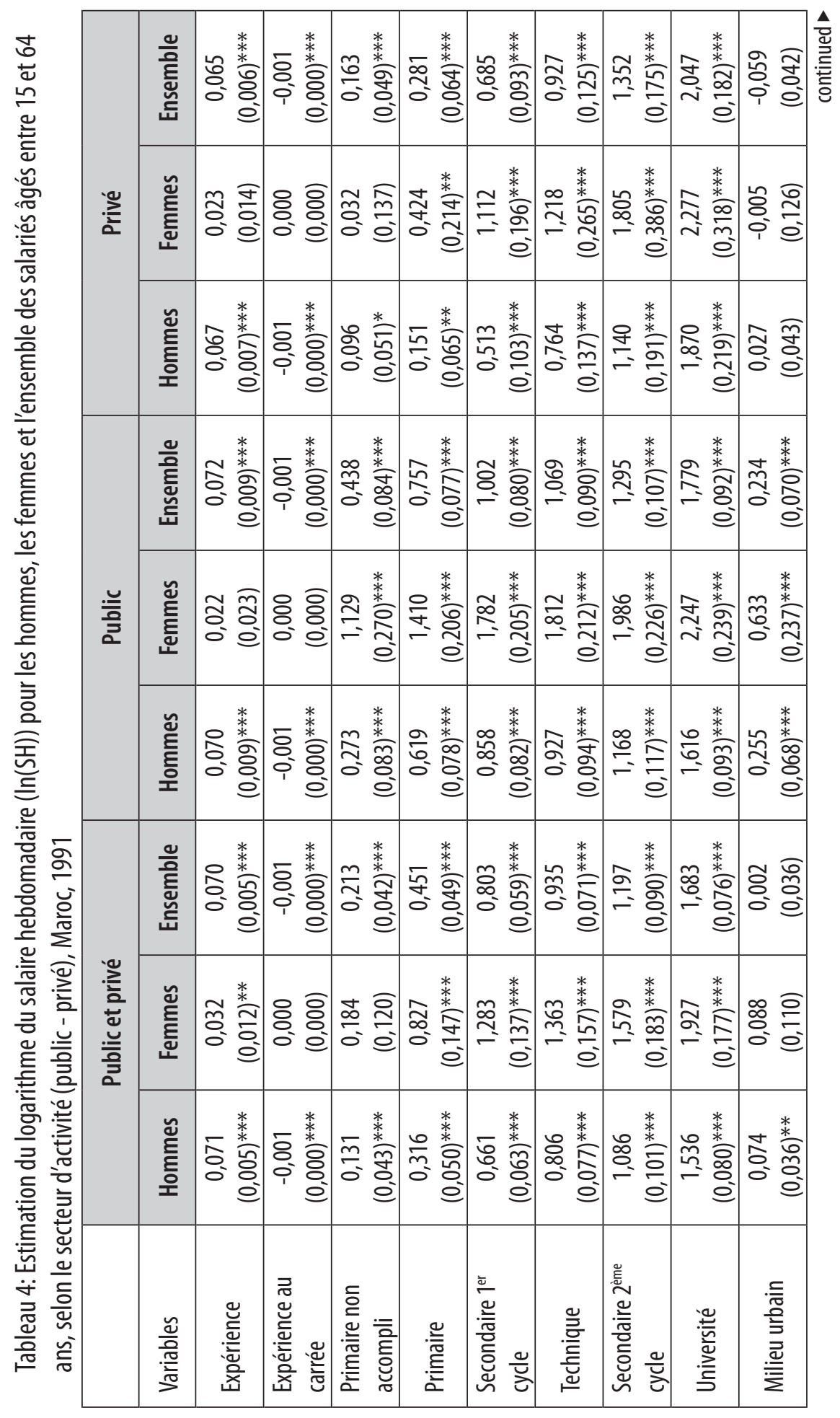




\begin{tabular}{|c|c|c|c|c|c|c|c|c|c|c|c|c|}
\hline 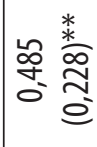 & & $\begin{array}{ll}\hat{n} & \bar{\theta} \\
0 & 0 \\
0 & 0\end{array}$ & 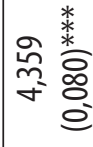 & ڤ્ & : & & 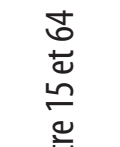 & & & $\begin{array}{l}0 \\
0 \\
0 \\
0\end{array}$ & 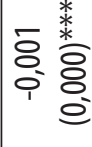 & s: \\
\hline 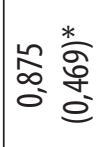 & & \begin{tabular}{cc}
$\nwarrow$ \\
\multirow{2}{*}{} \\
0 \\
0
\end{tabular} & 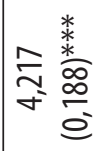 & I & 商 & & 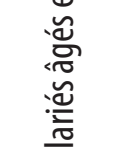 & : & & 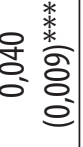 & $\begin{array}{l}\overline{2} \\
0 \\
0 \\
0 \\
0\end{array}$ & s: \\
\hline 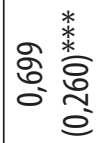 & & 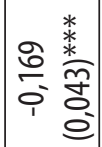 & 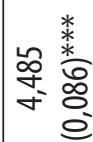 & $\Xi$ & 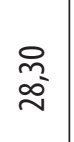 & & 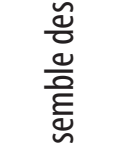 & & 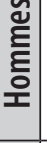 & $\begin{array}{l}5 \\
5 \\
5 \\
0\end{array}$ & 离 & \\
\hline 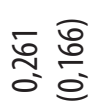 & & & 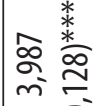 & ळి & 帝 & & & & $\underline{w}$ & : & $1 \stackrel{0}{0}$ & \\
\hline 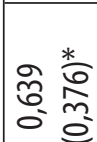 & & & 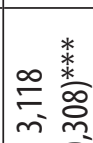 & $\stackrel{\infty}{=}$ & 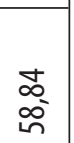 & 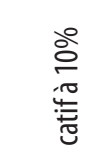 & 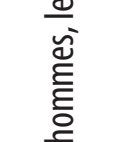 & $\frac{\breve{\underline{\underline{3}}}}{\overline{2}}$ & षे & & & \\
\hline & & & 尌 & 5 & 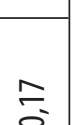 & $\begin{array}{l}\overline{\overline{\underline{E}}} \\
\overline{\underline{\underline{m}}}\end{array}$ & & & 호 & $\stackrel{0}{0}$ & $\begin{array}{l}\overline{5} \\
0 \\
0 \\
i \\
\end{array}$ & \\
\hline & & & $\bar{e}$ & & & & Е & & ״ำ & . & - 娄 & \\
\hline & $\stackrel{\infty}{\simeq} \underset{7}{\not}$ & & $\approx \widetilde{3}$ & $\mathbb{F}$ & $\Xi$ & 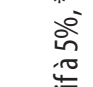 & 胥 : & & 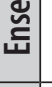 & 过 & : & \\
\hline$e^{-}$ & 08 & & $\sigma^{+} \stackrel{8}{\varrho}$ & & & & 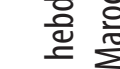 & 童 & ๗ & 。㐘 & б & $=\Omega^{*}$ \\
\hline & $g=$ & & 类 & $\bar{s}$ & $n$ & & 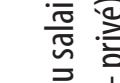 & $\frac{. \underline{\underline{n}}}{\bar{z}}$ & $\overline{\underline{L}}$ & 5 & (1) & \\
\hline & & 0 & $m$ & & & & & & $\check{\xi}$ & & 楚 & $=\bar{\infty}^{*}$ \\
\hline & 凅类 & $\frac{2}{2}$ & 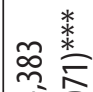 & గ్రిగ & $\stackrel{0}{\approx}$ & & $\frac{\overline{0}}{\bar{y}}$ & & 우 & 0 & e & \\
\hline & & & 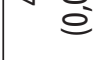 & & & & & & & & & \\
\hline 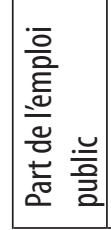 & 旁 & 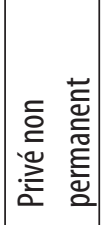 & 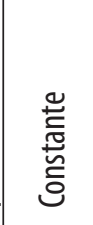 & 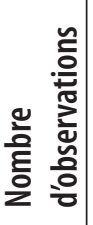 & $\approx$ & 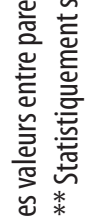 & 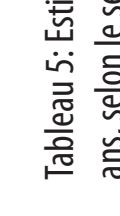 & & 然 & 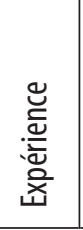 & 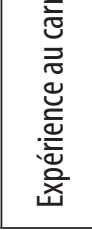 & 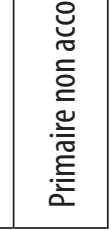 \\
\hline
\end{tabular}




\begin{tabular}{|c|c|c|c|c|c|c|c|c|c|c|c|c|c|}
\hline 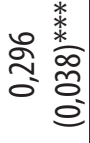 & 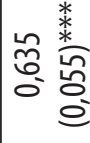 & 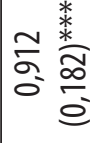 & 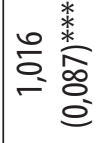 & 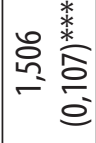 & 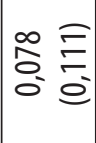 & 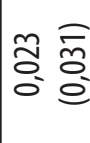 & 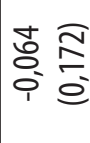 & ' & 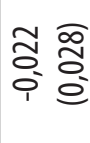 & 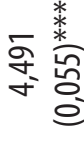 & 衣 & \begin{tabular}{l}
$\bar{\infty}$ \\
\multirow{J}{\pm}{}
\end{tabular} & \\
\hline 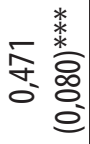 & 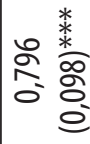 & 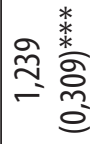 & 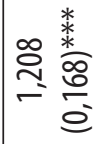 & 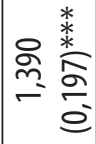 & 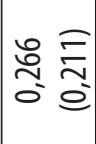 & 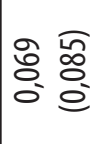 & 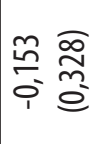 & 1 & 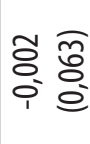 & 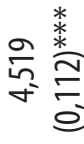 & ஜిరి & $\begin{array}{l}8 \\
\tilde{7}\end{array}$ & \\
\hline 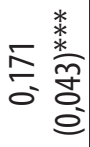 & 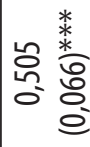 & 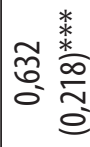 & 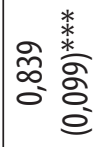 & 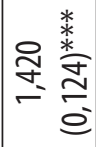 & 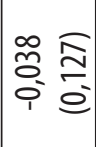 & 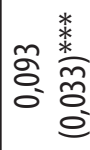 & 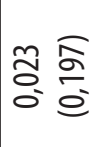 & I & 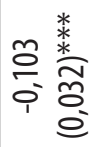 & 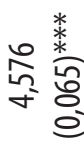 & 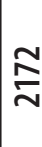 & $\stackrel{\simeq}{\approx}$ & \\
\hline 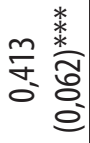 & 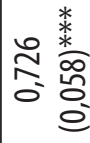 & 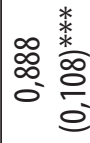 & 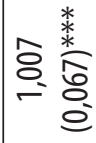 & 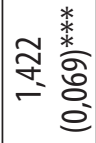 & 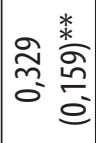 & 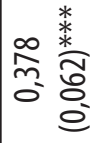 & 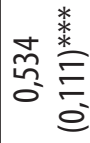 & 1 & I & 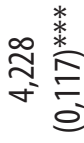 & ஃ & $\begin{array}{l}\tilde{Z} \\
\stackrel{\sim}{*}\end{array}$ & \\
\hline 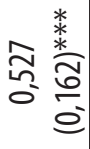 & 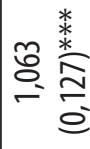 & 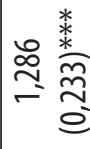 & 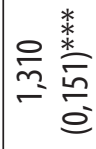 & 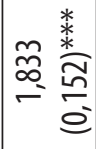 & 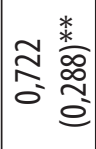 & 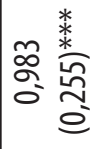 & 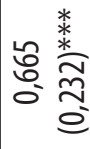 & ' & I & 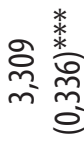 & $\tilde{\sim}$ & 읏 & 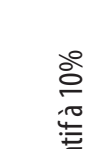 \\
\hline 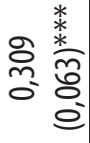 & 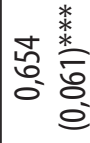 & 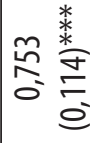 & 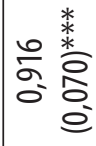 & $\frac{{ }^{*}}{\stackrel{*}{*}}$ & 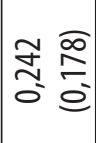 & 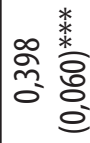 & 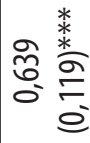 & ' & ' & 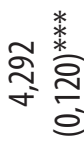 & กิ & $\begin{array}{l}20 \\
\text { 守 }\end{array}$ & 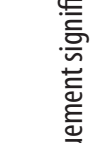 \\
\hline 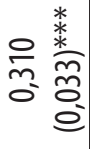 & 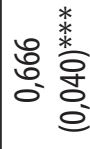 & 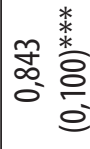 & 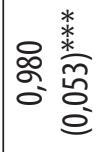 & 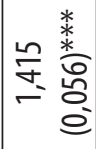 & 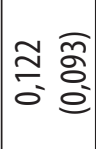 & 苍 & 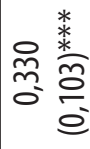 & 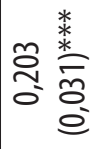 & $\begin{array}{ll} & 0 \\
0 & 0 \\
0 & 0 \\
0 & 0\end{array}$ & 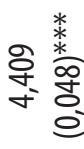 & ‡ & $\frac{\mathfrak{F}}{\mathscr{J}}$ & 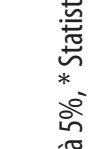 \\
\hline 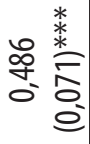 & 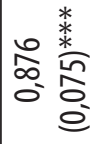 & 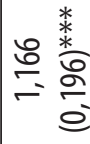 & 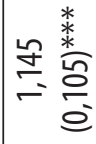 & 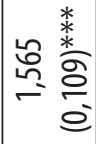 & 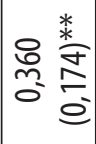 & $\begin{array}{ll}\bar{E} & \infty \\
\bar{\sigma} & 0 \\
0\end{array}$ & 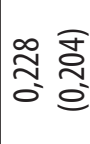 & 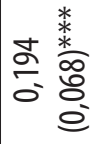 & 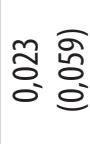 & 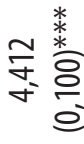 & $\approx$ & $\begin{array}{l}\infty \\
\text { ஐ̇ }\end{array}$ & 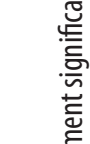 \\
\hline 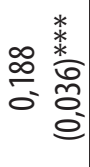 & 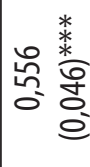 & 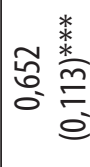 & 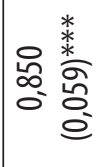 & 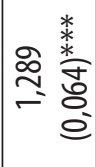 & 능 & $\begin{array}{ll}\infty & \frac{*}{*} \\
& \frac{*}{\infty} \\
& 0 \\
\end{array}$ & 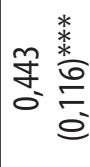 & 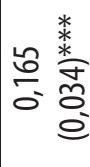 & 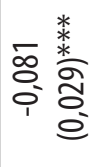 & 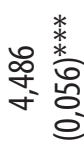 & & $\begin{array}{l}\infty \\
\text { rn} \\
\vartheta\end{array}$ & 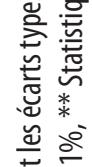 \\
\hline 离 & 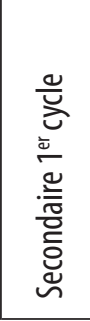 & 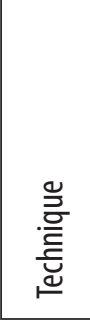 & 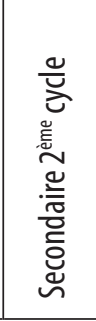 & 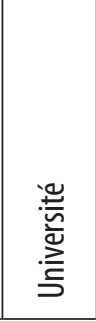 & 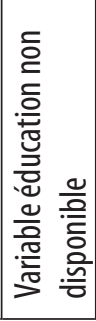 & 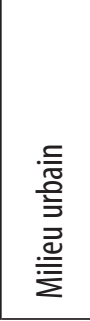 & 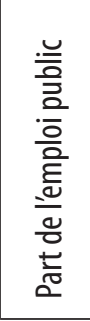 & $\frac{\frac{.}{0}}{\bar{a}}$ & 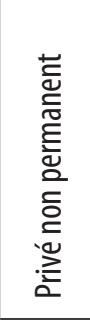 & 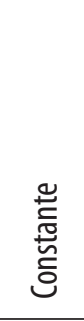 & 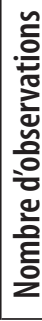 & $\approx$ & 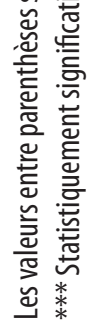 \\
\hline
\end{tabular}


Il apparait donc, à partir de notre analyse, que l'évolution des écarts de salaire entre les hommes et les femmes au Maroc s'était réduite relativement peu dans le privé mais considérablement dans le public. Ceci était dû d'un côté, aux efforts déployés par le gouvernement marocain dans le cadre de l'allègement de l'écart aussi bien dans le public que dans le privé, et de l'autre, à l'avancée enregistrée au niveau de l'éducation des femmes travaillant dans le public.

Tableau 6: Evolution de l'écart salarial entre les hommes et les femmes au Maroc, $1991-1999$

\begin{tabular}{|l|c|c|c|c|c|c|}
\hline & \multicolumn{2}{|c|}{ Total } & \multicolumn{2}{c|}{ Public } & \multicolumn{2}{c|}{ Privé } \\
\hline & 1991 & 1999 & 1991 & 1999 & 1991 & 1999 \\
\hline $\operatorname{In}(\mathrm{SHH})-\operatorname{In}(\mathrm{SHF})$ & $34,6 \%$ & $30,9 \%$ & $31,5 \%$ & $21,8 \%$ & $37,5 \%$ & $33,5 \%$ \\
\hline Ecart de salaire expliqué & $-1,1 \%$ & $1,5 \%$ & $-5,4 \%$ & $-13,1 \%$ & $0,8 \%$ & $5,9 \%$ \\
\hline Ecart de salaire inexpliqué & $35,7 \%$ & $29,4 \%$ & $36,9 \%$ & $34,9 \%$ & $36,7 \%$ & $27,6 \%$ \\
\hline
\end{tabular}

Figure 4: Ecart du salaire hebdomadaire par sexes, Maroc, 1991-1999

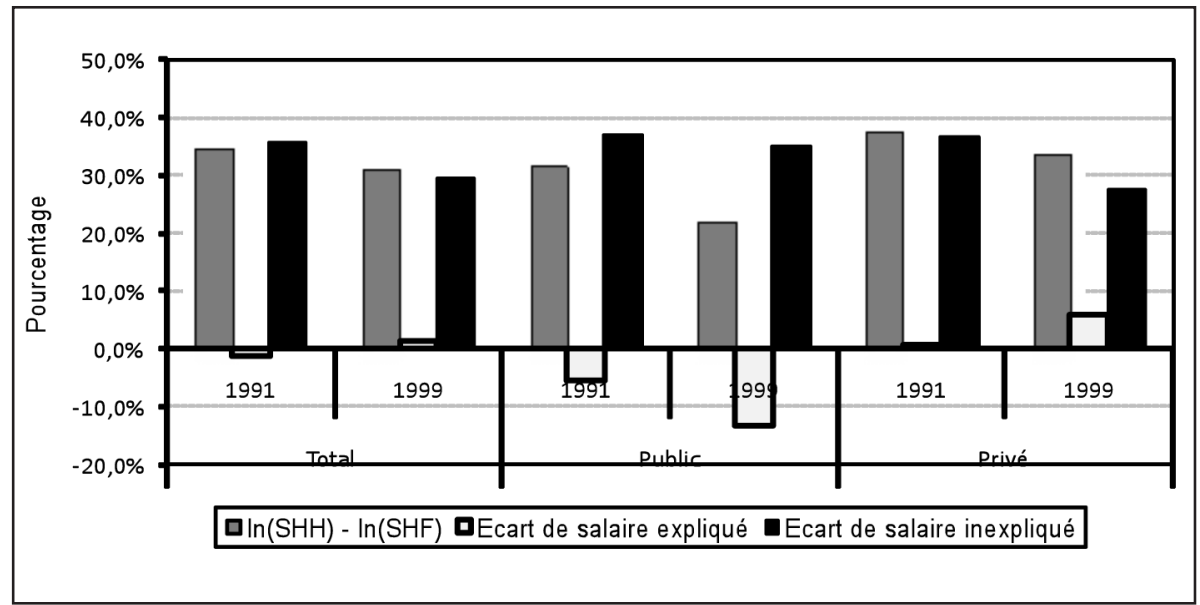

\section{Conclusions}

Malgré les nombreuses études faites au sujet de la discrimination, ce problème reste toujours au cœur des travaux de recherche accomplis aussi bien par les économistes que par les sociologues.

Nous avons essayé dans ce papier d'étudier l'écart salarial entre les hommes et les femmes au Maroc Urbain. Nous avons utilisé les méthodes de décompositions d'Oaxaca et Ransom et celle de Neumann et Oaxaca. 
Les estimations ont révélé que les femmes sont rémunérées 35 et $31 \%$ en moins que les hommes et ce successivement pour les périodes I99I et I999. Les différences de caractéristiques entre les deux sexes, dont principalement la différence des dotations en capital humain, explique toute seule une proportion très réduite de l'écart (-I,I\% en I99I et I,5\% en 1999). La proportion négative traduit en quelque sorte une avancée pour les femmes puisqu'il s'agit ici d'une différence qui peut être soit positive soit négative. Ayant obtenu un écart expliqué négatif en I99I voudrait dire qu'en termes d'aptitudes en capital humain, les femmes sont plus qualifiées que leurs homologues masculins. Ces écarts négatifs se confirmeront dans le secteur public en I99I $(-5,4 \%)$ et en $1999\left(-{ }_{3}, \mathrm{I} \%\right)$. Ceci confirme que les femmes, et surtout celles travaillant dans le public, ont des caractéristiques, essentiellement en terme d'éducation, plus importantes que les hommes ce qui leur donne un avantage partiel en terme de rémunération.

Les résultats montrent également que la discrimination salariale dans le public a régressé de io points entre I99I et I999. Alors que dans le public, la diminution n'était que de 4 points uniquement. Ceci pourrait être expliqué par le fait que les efforts déployés dans le secteur public pour éradiquer toutes formes de discrimination contre les femmes, ont trouvé leur compte. En effet, ces efforts se sont clairement concrétisés par une baisse de la discrimination le moins que l'on puisse dire c'est qu'elle est encourageante. Pour ce qui est du privé, beaucoup d'efforts restent à faire.

En fin de compte, il ne faut guère manquer d'insister sur le fait que nos investigations continuent dans le sens d'un développement plus approfondi du phénomène étudié afin de, tout d'abord, poursuivre l'analyse pour la rendre plus d'actualité. En effet, dans l'espoir d'avoir les données relatives à la dernière Enquête Nationale sur les Niveaux de Vie des Ménages au Maroc qui a eu lieu en 2006/07, nous essayerons de prolonger notre étude pour couvrir toute la période $1990-2006$. Ensuite, nous comptons développer une technique consistante ayant l'objectif de surmonter le problème d'indisponibilité d'information relative au nombre d'heures travaillées dans l'échantillon de I999. Enfin, nous essayerons de reprendre le travail de décompositions tout en répartissant les écarts salariaux entre les hommes et les femmes par tranches d'âge. Nous considérerons, principalement, les jeunes âgés entre 15 et 24 et les adultes pour le reste. Ceci pourrait révéler quelques observations pouvant être intéressantes quant à la discrimination au sein des jeunes salariés.

En conséquence, et comme recommandations, nous présenterons quelques unes dans ce qui suit. 


\section{Recommandations}

A la lumière des investigations faites tout au long de cette étude, il s'avère important de mettre en place un certain nombre de mesures à entreprendre afin de contribuer, même partiellement, à la résolution du problème de discrimination. Dans ce qui suit quelques unes:

- Favoriser le développement de la formation, de l'éducation et du niveau culturel non pas seulement dans la société marocaine mais aussi dans les sociétés maghrébines. En effet, le développement du niveau éducatif pourrait améliorer le sort des femmes qui sauront leurs devoirs et leurs droits et pourront facilement s'incarner dans les composantes de la société civile. Ce développement est suscité surtout dans les domaines qui fournissent les meilleures qualifications demandées par le marché. Il est évident que les hommes soient eux aussi confrontés à ce problème. Cependant, les femmes ayant plus d'exigences sociales (mariage précoce et éducation des enfants) qui les conduisent à interrompre leur scolarisation ou à arrêter leur travail, sont victimes de la dévalorisation de leurs diplômes et de leur savoir faire.

- Penser à “ la discrimination positive " afin de corriger les inégalités socio-économiques. En effet, il s'agit d'instituer des inégalités pour promouvoir l'égalité. Cette forme de discrimination consiste à orienter vers les plus démunis une part accrue des prestations sociales et des dépenses publiques et ce par la mise en place d'un Etat-providence désormais sélectif, qui choisit les ayants droits et les usagers en fonction de critères socio-économiques. Sur le plan du marché du travail, les femmes discriminées peuvent bénéficier de ce genre de mécanismes si nous les orienterons vers la demande féminine de l'emploi d'un côté et vers les moins rémunérées de l'autre. Il sera ainsi primordial de commencer par identifier " une population cible" afin de pouvoir agir quand il faut et comme il le faut.

- Développer les travaux susceptibles d'alimenter une base de données visualisant l'état des lieux des pratiques discriminatoires dans le pays. Ladite base de données pourrait être établie moyennant des enquêtes périodiques qui seront faites et analysées par des spécialistes qui pourront mettre en place des propositions de lutte contre les pratiques discriminatoires mieux adaptées à la réalité socio-économique du pays. Ceci servira pour sensibiliser les gouvernements à ce type de problèmes fréquemment rencontrés sur le marché du travail. 
Il sera tout de même opportun de développer une sorte d'entraide féminine à travers les lobbies et les associations qui auront pour tâche l'amélioration de la condition de la femme et son intégration dans une économie confrontée de plus en plus à la mondialisation. Lesdites associations seront un outil d'action et de sensibilisation des femmes surtout les moins scolarisées vu qu'elles représentent la population féminine la plus vulnérable.

- Fournir un effort double, de la part des femmes, pour montrer que leur accession n'est due qu'à leurs aptitudes professionnelles. A ce niveau, il faut inculquer aux femmes dès leur premier âge la confiance en soi. Lintimidation et le sentiment d'être inférieures aux hommes comptent parmi les facteurs essentiels dans les pratiques discriminatoires contre elles. C'est pour cela que nous voyons dans la confiance en soi un facteur principal pouvant contribuer à l'amélioration de la situation des femmes sur le marché du travail et par conséquence dans la société.

- Favoriser l'implication des femmes dans les processus de prise de décision afin de créer un environnement genre plus équitable dans les sphères aussi bien économique que sociale. Cette implication serait possible via une participation accrue des femmes dans la vie politique. En effet, il a été conclu qu'une participation politique féminine plus active pourrait être la clé pour accomplir le changement. Le paradigme traditionnel du genre n'est pas uniquement un aspect important de la législation mais aussi de l'implication des femmes dans la vie politique et dans tout autre niveau de prise de décision. A ce niveau, d'énormes efforts restent à déployer pour garantir aux femmes un meilleur sort politique.

- Uniformiser le cadre législatif afin que les droits constitutionnels des femmes soient cohérents avec la législation ordinaire. Il faut remarquer à ce niveau que certaines dispositions légales ont échoué dans la réalisation de cet équilibre. C'est pour cela que les lois et les réglementations du travail doivent être synchronisées avec le nouveau modèle du développement économique et social qui règne dans la région. C'est dans ces conditions que sera créée une meilleure incitation pour la création d'emplois dans le secteur privé au sein d'un environnement moins discriminatoire. D'ailleurs, un certain nombre de règlements sur le marché du travail contribuent à l'élévation du coût de la main-d'œuvre féminine par rapport à celle masculine. 
- Favoriser l'accès des femmes à la sphère publique qui était jusqu'à une date récente quasi-masculine. Pour atteindre un tel objectif, les gouvernements sont invités à multiplier les investissements dans l'infrastructure de base (transport, eau, télécommunication, etc.) qui pourrait améliorer les horizons de la femme et lui permet de mieux arbitrer entre responsabilités professionnelles d'un côté et familiales de l'autre (un service efficace de garde d'enfants pourrait être essentiel). De cette manière, les femmes accèderont aux postes clés, générateurs de puissance, d'influence et de patronage. Cet accès permettra au genre féminin d'occuper sa vraie place dans la société et de mettre fin à sa propre vulnérabilité. Ceci étant, la question qui doit être posée à ce niveau: dans quelle mesure la femme, une fois atteignant ces postes dites clés, est capable d'agir en faveur de ses compatriotes contre les pratiques discriminatoires?

La question que nous venons d'expliciter mérite d'être analyser plus profondément surtout qu'elle ouvre les horizons devant les chercheurs afin de se demander comment sera perçu l'appel à l'égalité des genres dans une société comme la nôtre? 


\section{References}

Aigner, D. \& G. Cain. (1977), "Statistical Theories of Discrimination in Labor Market", Industrial and Labor Relations Review, Vol 30, n 2, pp. I75-187.

Arrow. K. (1973), "The theory of discrimination", in Discrimination in labor markets, O. Ashenfelter and A Rees eds, Princeton University Press.

Assaad, R. \& S. Zouari. (2002), "The Timing of Marriage, Fertility and Female Labor Force Participation in Urban Morocco", Papier presenté à la

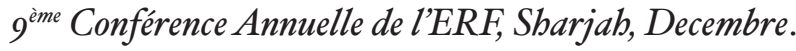

Barbezat, D. (1987), "Salary Differentials by Sex in the Academic Labor Market", The fournal of Human Resources, Vol. 22, pp. 423-428.

Bayet, A. (1996), "Léventail des Salaires et ses Déterminants", Données Sociales.

Becker, G. (1957), "The Economics of Discrimination”, Second Edition, University of Chicago Press, Chicago.

Bertrand, M. \& K. F. Hallock. (200I), "The Gender Gap in Top Corporate Jobs", Industrial and Labor Relations Review, Vol. 55, n I, pp. 3-19.

Biddle, J. \& D. S. Hamermesh. (1998), "Beauty, Productivity and Discrimination: Lawyers' Looks and Lucre”, Fournal of Labor Economics, Vol. I6, n'I, pp. 172-20I.

Blau, F. D. \& L. M. Kahn. (1999), "Analyzing the Gender Pay Gap", The 2uarterly Review of Economics and Finance, Vol. 39, n 5, pp. $625^{\circ} 646$.

Blinder, A. S. (1973), "Wage Discrimination: Reduced Form and Structural Estimates", The fournal of Human Resources, Vol 8, n ${ }^{\circ}$, pp. 436455 .

Combarnous, F. (I996), "Discrimination du Marché du Travail Urbain: Le Cas d'Abidjan en Côte d'Ivoire", Document de travail, CED, Université Montesquieu Bordeaux 4, France.

Cotton, J. (1988), "On the Decomposition of Wage Differentials", Review of Economics and Statistics, Vol. 70, pp. 236-243.

Curraize. Y. et Hugounenq. R. (2004), "Inégalités de salaires entre femmes et hommes: le poids de la discrimination", Problèmes économiques, $\mathrm{n}^{\circ} 2857$, pp $4 \mathrm{I}^{-} 48$.

El-Hamidi F. et Mona Said. (2005), "Wage Inequality by Education and Gender in MENA: Contrasting the Egyptian and Moroccan Experiences in the 1990's", Conference Paper ERF website:

bttp://wwwerf.org.eg/cms.php?id=publication_detailsopublication_id=506 
Ferber, M. A. \& C. A. Green. (I982), "Traditional or Reverse Sex Discrimination? The Case of a Large Public University", Industrial and Labor Relations Review, Vol. $35, \mathrm{n}^{\circ} 4$, pp. 550-564.

Gander, J. P. (1997), "Gender-Based Faculty-Pay Differences in Academe: A Reduced Form Approach”, Journal of Labor Research, Vol. 18, n³, pp. 451-461.

Hashimoto, M. (198I), "Firm Specific Human capital as a shared investment", The American Economic Review, June, Vol 71, n³ ${ }^{\circ}$, pp.475-482.

Heckman, J. (1979), "Sample selection bias as a specification error", Econometrica, Vol. 47, n 1, pp. 153-161.

Kraus, V. \& Y. P. Yonay. (2000), “The Effect of Occupational Sex Composition on the Gender Gap in Workplace Authority”, Social Science Research, Vol. 29, pp. 583-605.

(Lazear et Rosen (1990), Lewbel, A. (2002), "Selection Model and Conditional Treatment Effects, Including Endogenous Regressors", mimeo, Boston College.

LHéritier, J. L. (I992), "Les déterminants du salaire”, Economie et Statistique, $\mathrm{n}^{\circ} 257$.

Meurs, D. \& S. Ponthieux. (2000), "Une mesure de la discrimination dans l'écart de salaire entre hommes et femmes", Economie et Statistique, n 337-338, pp. $135-152$.

Meurs, D. \& S. Ponthieux. (2004), "Les écarts de salaires entre les femmes et les hommes en Europe. Effet de structure ou discrimination ?" Revue de l'OFCE, $\mathrm{n}^{\circ} 90$, pp.153-192.

Mincer, J. (1974), "Schooling, experience and earnings", NY: Columbia University Press.

Neumann S. \& R.L Oaxaca (2004), "Gender versus ethnic wage differentials Among Professionals: evidence from Israel" Annales d'économie et de statistique, $\mathrm{n}^{\circ} 71-72$, pp.267-292.

Oaxaca R. L. (1973), "Male-female wage differentials in urban labor markets", International economic review, Vol 14, n 3 pp. 693-709.

Oaxaca R. L. \& M. R. Ransom (I988), "Searching for the effect of unionis on the image of union and nonunion workers", Journal of Labour Research, Vol 9, pp. 139-148.

Oaxaca R. L. \& M. R. Ransom (I994), "On discrimination and the decomposition of wage differentials", Journal of Econometrics, Vol.61, n ${ }^{\circ} 1, \mathrm{pp} .5-21$.

Phelps, E. (I972), "The Statistical Theory of Racism and Sexism”, American Economic Review, Vol 62, n 4, pp. 659-661.

Ransom, M. R. \& S. B. Megdal. (I993), "Sex Differences in the Academic Labor Market in the Affirmative Action Era", Economics of Education Review, Vol $12, n^{\circ} 1$, pp. 21-43. 
Riboud M. (1978), “Accumulation du capital humain”, Paris, Economica.

Serumaga-Zake, P. AE. \& W. Naudé. (200I), "Labor Market Discrimination in the North West Province of South Africa", Development Southern Africa, Vol I8, n 5 , pp. 671-682. 

